\title{
Prostate-specific membrane antigen (PSMA)-based imaging in localized and advanced prostate cancer: a narrative review
}

\author{
Ivan de Kouchkovsky ${ }^{1}$, Rahul Aggarwal ${ }^{1}$, Thomas A. Hope ${ }^{1,2}$ \\ ${ }^{1}$ Helen Diller Family Comprehensive Cancer Center, University of California San Francisco, San Francisco, CA, USA; ${ }^{2}$ Department of Radiology \\ and Biomedical Imaging, University of California San Francisco, San Francisco, CA, USA \\ Contributions: (I) Conception and design: All authors; (II) Administrative support: None; (III) Provision of study materials or patients: None; (IV) \\ Collection and assembly of data: None; (V) Data analysis and interpretation: None; (VI) Manuscript writing: All authors; (VII) Final approval of \\ manuscript: All authors. \\ Correspondence to: Thomas A. Hope. 185 Berry Street, Suite 350, San Francisco, CA 94107, USA. Email: Thomas.Hope@ucsf.edu.
}

\begin{abstract}
Combined molecular and morphologic imaging modalities have emerged in recent years as an alternative to conventional imaging in prostate cancer (PC). In particular, novel prostate-specific membrane antigen (PSMA) radiotracers have demonstrated increased sensitivity and specificity for the initial staging of men with clinically localized PC, as well as for PC detection in the setting of biochemical recurrence (BCR). Molecular imaging is increasingly used to guide treatment decisions in these patients-though its impact on survival has yet to be established. Improved PC detection in men with BCR has also helped to identify a subset of patients with oligometastatic disease. The optimal management of oligometastatic PC and the role of metastasis-directed therapies (MDT) are the subjects of ongoing studies. In comparison to clinically localized or biochemically recurrent PC, the role of molecular imaging in men with advanced disease is less established. In metastatic castration-resistant PC (mCRPC), PSMA-based imaging has primarily been investigated as a companion diagnostic tool to predict and monitor response to PSMA-targeted radioligand therapy (RLT). More recent efforts have focused on using molecular imaging to monitor treatment response to conventional chemohormonal therapies. However, despite promising early results, several barriers remain to the widespread use of PSMA-based imaging in metastatic PC: temporary flares in PSMA uptake have been described in a subset of patients after initiation of therapy, and the underlying mechanism and clinical implications of this phenomenon are still poorly understood. Furthermore, whereas PSMA is invariably expressed in hormone-sensitive PC, loss of PSMA expression is increasingly recognized in a subset of mCRPC patients with aggressive disease. Although this may limit the use of PSMA-based imaging as a standalone modality in advanced PC, loss of PSMA uptake may also provide non-invasive and clinically relevant molecular insight on patients' underlying tumor biology.
\end{abstract}

Keywords: Prostate-specific membrane antigen (PSMA); glutamate carboxypeptidase II; positron emission tomography (PET); prostatic neoplasms/diagnostic imaging

Submitted Jul 01, 2020. Accepted for publication Oct 30, 2020.

doi: $10.21037 /$ tau-20-1047

View this article at: http://dx.doi.org/10.21037/tau-20-1047 


\section{Introduction}

Prostate cancer (PC) is the most common cancer in men in the United States (US), with over 170,000 estimated cases and 31,000 deaths in 2019 alone (1). Imaging plays a crucial role in the diagnosis and management of $\mathrm{PC}$ as it progresses from clinically localized disease to metastatic castrationresistant PC (mCRPC). Conventional imaging modalities used in PC consist of cross-sectional imaging via computed tomography (CT) or magnetic resonance imaging (MRI), and ${ }^{99 \mathrm{~m}} \mathrm{Tc}-\mathrm{methylene}$ diphosphonate radionuclide bone scintigraphy. Though widely available, these modalities have important limitations in the imaging and management of PC. Both CT and MRI rely on size and anatomic abnormalities to detect nodal and visceral metastases; early nodal involvement may be missed (2), leading to the under-staging of patients with clinically localized disease or biochemical recurrence (BCR). Moreover, they have poor sensitivity and specificity for osseous metastases. Bone scintigraphy, which detects reactive osteoblastic activity, similarly lacks specificity for PC and often lags behind treatment responses or disease progression (3).

In more recent years, combined molecular and morphologic imaging modalities have emerged as an alternative to conventional imaging for the detection and monitoring of PC. Additionally, they are increasingly recognized for their ability to provide a non-invasive assessment of underlying PC biology. Here we review selected novel imaging modalities in PC, with a focus on prostate-specific membrane antigen (PSMA) targeted imaging. We discuss the strength and limitations of molecular-based imaging compared to conventional modalities across the different stages of PC, as well as the impact of novel radiotracers on the PC treatment landscape. We present the following article in accordance with the Narrative Review reporting checklist (available at http:// dx.doi.org/10.21037/tau-20-1047).

\section{Methods}

Identification of relevant studies occurred by search of the Cochrane Library, Google Scholar, and MEDLINE indexed journals published in the English language from January $1^{\text {st }}$, 2010 to September $1^{\text {st }}, 2020$. A search of the grey literature from September $1^{\text {st }}, 2018$ through September $1^{\text {st }}, 2020$ was also performed to identify relevant abstracts and conference proceedings. Search terms included combinations of the following key words: prostatic neoplasm, PET, PSMA or
FOLH1 protein, choline, and fluciclovine. Results were filtered for observational studies, clinical trials, systematic reviews or meta-analyses. Relevant articles were then assessed via titles and abstracts. Inclusion of prospective studies and meta-analyses was prioritized over retrospective analyses.

\section{Molecular imaging modalities in PC}

${ }^{18} \mathrm{~F}$-fluorodeoxyglucose $\left({ }^{18} \mathrm{FDG}\right)$ positron emission tomography (PET) imaging plays a limited role in the detection of clinically localized or biochemically recurrent $\mathrm{PC}(4,5)$, and various alternative tracers have been investigated over the past several decades. ${ }^{11} \mathrm{C}$ - and ${ }^{18}$ F-radiolabelled choline-a phospholipid precursor selectively taken up by rapidly dividing cancer cells-were among the first non-FDG PET tracers studied in patients with BCR. An early study of ${ }^{11} \mathrm{C}$-choline PET/CT showed improved sensitivity over conventional imaging for the detection of pelvic node recurrences in patients with BCR (6), and it received FDA approval for this indication in 2012. However, the sensitivity of ${ }^{11} \mathrm{C}$-choline drops off significantly in patients with low serum prostate-specific antigen (PSA) levels (7) — who are most likely to benefit from salvage radiation therapy (RT). The very short halflife (20 minutes) and the resulting need for on-site cyclotron production have also limited its wide-scale implementation. In comparison the diagnostic performance of ${ }^{18} \mathrm{~F}$-choline appears to be similar to that of ${ }^{11} \mathrm{C}$-choline $(8,9)$, while its longer half-life (110 minutes) allows off-site production. Although it was never approved in the US, this tracer is still routinely used in some parts of the world.

Fluciclovine is an ${ }^{18} \mathrm{~F}$-labelled analog of L-leucine selectively taken up by amino acid transporters upregulated on the surface of PC cells. Unlike ${ }^{18}$ F-FDG or ${ }^{18}$ F-choline it has negligible urinary excretion, which allows for improved detection of locoregional disease. An early study comparing fluciclovine to ${ }^{11} \mathrm{C}$-choline in patients with BCR showed improved specificity and detection rates with fluciclovine PET/CT [positive predictive value (PPV) of 97\% versus 90\%] (10). Although the performance of both modalities was dependent on PSA level, the sensitivity of fluciclovine was consistently higher than that of ${ }^{11} \mathrm{C}$-choline. These results should be interpreted with caution, as pathologic confirmation was available only in a minority of patients and a lower than conventional dose $(3.4 \mathrm{MBq} / \mathrm{kg})$ of ${ }^{11} \mathrm{C}$-choline was used. A subsequent meta-analysis of 9 studies and 363 patients with BCR undergoing fluciclovine 
imaging showed a pooled sensitivity and specificity of $88 \%$ and $73 \%$, respectively (11). As of 2016 , fluciclovinePET/CT is FDA approved for the diagnosis of recurrent PC in patients with BCR. Yet as with ${ }^{18} \mathrm{~F}$ - or ${ }^{11} \mathrm{C}$-choline, the sensitivity of fluciclovine in patients with low serum PSA remains suboptimal $(12,13)$. Similarly, it has not been shown to significantly improve the diagnostic performance of conventional imaging in the initial staging of PC (14).

PSMA is a transmembrane protein produced by epithelial cells in the proximal renal tubules, salivary glands, small bowel and prostate gland (15). It is upregulated in PC cells by up to 1,000 -fold (15) and provides an ideal target for PC detection. Ligand internalization upon binding to the extracellular portion of PSMA further improves tumor visualization. Though initial PSMA-targeted monoclonal antibodies were characterized by long circulating half-lives and high levels of background activity, these limitations were largely overcome with the advent of small-molecule radiotracers. ${ }^{68} \mathrm{Ga}$-PSMA-HBED-CC $\left({ }^{68} \mathrm{Ga}\right.$-PSMA-11) is the most widely studied of these ligands, though related compounds such as ${ }^{18} \mathrm{~F}$-DCFPyL and the DOTAconjugated PSMA-617 tracer have also been investigated. Due to the relatively short half-live of ${ }^{68} \mathrm{Ga}$ (68 minutes), Ga-based radiotracers generally require local productionthrough either a Ga generator or an on-site cyclotron (16). On the other hand, the longer half-life of ${ }^{18} \mathrm{~F}$ (110 minutes) allows for centralized production and widescale distribution of ${ }^{18} \mathrm{~F}$-labelled tracers such as ${ }^{18} \mathrm{~F}$-DCFPyL. Non-renal clearance of ${ }^{18} \mathrm{~F}$-labelled radiotracers could theoretically improve detection of locoregional recurrence, though current evidence suggests that the diagnostic performance of ${ }^{68} \mathrm{Ga}$ - and ${ }^{18} \mathrm{~F}$-labeled PSMA radiotracers is comparable (as discussed below). Since the first report of ${ }^{68} \mathrm{Ga}-$ PSMA-11 PET/CT in PC patients in 2013 (17), the field of PSMA-based imaging has seen exponential growth. In the remainder of this review, we discuss the existing evidence behind the use of PSMA-targeted PET imaging in men with clinically localized, biochemically recurrent, and advanced PC.

\section{PSMA PET for initial staging}

PSMA-based imaging has shown improved sensitivity and diagnostic accuracy in the initial staging of men with clinically localized intermediate-to-high-risk PC. In 2016 Maurer and colleagues retrospectively reviewed 130 patients with intermediate-to-high-risk PC who had undergone initial staging with both conventional (CT or MR) and
${ }^{68} \mathrm{Ga}$-PSMA-11 PET imaging prior to radical prostatectomy (RP) with pelvic lymph node dissection (PLND). Thirty-one percent of patients had lymph node metastases at the time of surgery, and ${ }^{68} \mathrm{Ga}$-PSMA-11 PET significantly outperformed conventional morphologic imaging with patient-based sensitivities of $65.9 \%$ versus $43.9 \%$, and diagnostic accuracies of $88.5 \%$ versus $72.3 \%$, respectively (18). A pooled analysis including 4 additional trials and a combined total of 266 patients confirmed these early results, reporting a sensitivity of $74 \%, \mathrm{PPV}$ of $85 \%$ and accuracy of $86 \%$ for extraprostatic disease (19).

Several recent prospective studies have further evaluated the diagnostic accuracy of PSMA-based imaging in clinically localized PC. van Kalmthout and colleagues performed ${ }^{68} \mathrm{Ga}$-PSMA-11 imaging in 103 patients with newly diagnosed intermediate-to-high-risk PC and negative bone scans. Extended PLND was performed in 94\% cases, $42.3 \%$ of which were found to have lymph node metastases. The patient-based sensitivity, specificity and PPV of ${ }^{68} \mathrm{Ga}$-PSMA-11 imaging were $41.5 \%, 90.9 \%$, and $77.3 \%$, respectively $(19,20)$. The OSPREY trial prospectively evaluated the diagnostic performance of ${ }^{18} \mathrm{~F}$-DCFPyL PET/CT in 252 men with clinically localized high-risk PC planned to undergo RP with PLND. ${ }^{18} \mathrm{~F}$-DCFPyL PET/CT identified nodal or metastatic disease in $14.7 \%$ and $10.7 \%$ of cases, respectively. After histopathologic confirmation, the node-based sensitivity and PPV of ${ }^{18} \mathrm{~F}$-DCFPyL were $31-42 \%$ and $78-91 \%$, respectively (21). Comparable node- and patient-based sensitivities of $34.7 \%$ and $41.2 \%$ were observed in another trial of ${ }^{18} \mathrm{~F}$-DCFPyL $\mathrm{PET} / \mathrm{CT}$ in patients with intermediate-to-high-risk PC undergoing RP with PLND (the SALT trial) (22). Finally, ${ }^{68} \mathrm{Ga}$-PSMA-11 PET imaging was directly compared to conventional imaging in a large multicenter randomized clinical trial of 302 men with newly diagnosed high-risk PC (ProPSMA). Using a composite reference standard of histopathologic, imaging, clinical and biochemical findings - with only $28 \%$ of patients undergoing pelvic node sampling - the 6-month incidence of pelvic or distant metastases was $30 \% .{ }^{68} \mathrm{Ga}-\mathrm{PSMA}-11 \mathrm{PET} / \mathrm{CT}$ significantly outperformed conventional imaging, with diagnostic accuracies of $92 \%$ and $65 \%$, sensitivities of $85 \%$ and $38 \%$, and specificities of $98 \%$ and $91 \%$, respectively (23). Importantly, selection for a higher risk population and lack of a confirmatory pelvic nodal dissection in most cases may have contributed to the higher sensitivity and PPV observed in ProPSMA compared to other studies of PSMA-based imaging in patients with clinically localized PC (20-23). 
Interestingly, the design of the ProPSMA trial allowed patients with 3 or fewer distant metastases on conventional imaging to undergo ${ }^{68} \mathrm{Ga}-\mathrm{PSMA}-11 \mathrm{PET} / \mathrm{CT}$. These results led to a significant management change (e.g., change in treatment intent, modality, extent of surgery or RT dose/volume) in $27 \%$ of cases (23). A comparable rate of management changes after ${ }^{68} \mathrm{Ga}$-PSMA-11 imaging was observed in an earlier prospective study by Roach and colleagues (24). As in BCR however, the impact of PSMA-based imaging on patient outcomes has yet to be determined. In light of this evidence gap, current European Urology Association (EUA) guidelines do not make any specific recommendation for the use of PSMA-based imaging for PC staging (25).

\section{PSMA PET in biochemically recurrent PC}

Early applications of PSMA PET/CT focused on detecting recurrent $\mathrm{PC}$ in men with a rising PSA after prior definitive therapy (Figure 1). In 2013, Afshar-Oromieh and colleagues described the use of ${ }^{68} \mathrm{Ga}$-PSMA-11 PET/CT in 37 patients with BCR. Recurrent PC was detected in $83.8 \%$ of patients in the entire cohort (median PSA level of $3.3 \mathrm{ng} / \mathrm{dL}$ ), and among $60 \%$ of patients with a PSA level $<2.2 \mathrm{ng} / \mathrm{dL}$ (17). A later series of 1,007 patients with BCR confirmed these results, with a detection rate of $67 \%$ among patients with a serum PSA $<2$ ng/dL (26).

Although histopathologic confirmation is rarely pursued in men with BCR, a 2019 meta-analysis of 256 patients included across 15 studies with pathologic correlation estimated a near-perfect per-patient sensitivity and PPV of $99 \%$ and $99 \%$, respectively (19). The results should be interpreted with caution, as the retrospective nature of most studies, and frequent use of preoperative imaging to guide the extend of PLND may have overestimated the diagnostic performance of ${ }^{68} \mathrm{Ga}$-PSMA-11. A more recent prospective study showed an overall detection rate of $75 \%$ - and $38 \%$ in those with PSA level less than $0.5 \mathrm{ng} / \mathrm{mL}$ (27). Lesion validation was performed in 223 of 635 patients (217 of which had a positive ${ }^{68} \mathrm{Ga}$-PSMA-11 PET/CT) using a composite reference standard of histopathologic, imaging and PSA follow-up. On a per-patient basis, the PPV of a positive scan was $92 \%$; among the $87(39 \%)$ patients who had undergone histopathologic confirmation, the per-patient PPV and sensitivity of ${ }^{68}$ Ga-PSMA-11 were $84 \%$ and $92 \%$, respectively. The diagnostic performance of ${ }^{18} \mathrm{~F}$-labeled PSMA tracers appears comparable to that ${ }^{68} \mathrm{Ga}$-PSMA-11, with preliminary results from the multicenter phase III CONDOR study showing a PPV of $85 \%$ to $87 \%$ among 208 men with BCR (median PSA $0.8 \mathrm{ng} / \mathrm{dL}$ ) undergoing ${ }^{18} \mathrm{~F}$-DCFPyL imaging (28), and a pooled detection rate of $81 \%$ in a recent meta-analysis of ${ }^{18}$ F-labeled PSMA PET/CT in BCR (29).

As with choline and fluciclovine, the performance of PSMA-based imaging remains dependent on serum PSA levels. However, ${ }^{68} \mathrm{Ga}$-PSMA-11 PET has shown improved sensitivity for recurrent $\mathrm{PC}$ in patients with low serum PSA, with one systematic review of 4,790 combined BCR patients reporting patient-based detection rates of $33 \%$ and $45 \%$ at PSA levels $<0.2$ and $0.2-0.49 \mathrm{ng} / \mathrm{mL}$, respectively (30). In line with this, a 2019 meta-analysis evaluating the diagnostic performance of PSMA- versus choline-based PET imaging in BCR showed a superior per-patient detection rate with PSMA-based imaging (78\% versus 56\%), primarily driven by improved PC detection at serum PSA levels less than $1 \mathrm{ng} / \mathrm{mL}$ (per-patient detection rates of $54 \%$ versus 27\% for PSMA and choline PET/CT, respectively) (31). Similarly, a more recent prospective single-center study of ${ }^{68} \mathrm{Ga}$-PSMA-11 and fluciclovine PET imaging in $50 \mathrm{BCR}$ patients with serum PSA levels less than $2.0 \mathrm{ng} / \mathrm{mL}$ showed superior detection rates with ${ }^{68} \mathrm{Ga}$-PSMA-11 (per-patient detection rate of $56 \%$ versus $26 \%$ with ${ }^{68} \mathrm{Ga}$-PSMA- 11 and fluciclovine PET/CT, respectively) (32) and a tumorto-background ratio up to 8 times higher than that of fluciclovine.

Improved PC detection in patients with BCR has significant implications for treatment planning: accurate disease localization may help to select patients who are most likely to benefit from salvage or metastasis-directed therapy (MDT), and conversely spare the toxicities of localized therapy in men with more advanced disease. The ability of PSMA-based imaging to detect recurrent PC at very low serum PSA levels is particularly appealing: in a post-hoc analysis of 270 patients with early BCR (serum PSA level $<1 \mathrm{ng} / \mathrm{dL}$ ) undergoing salvage radiation to the prostate bed and pelvic lymph nodes, ${ }^{68} \mathrm{Ga}$-PSMA-11 PET revealed one or more lesion outside of the planned radiation field in $19 \%$ of cases (33). Other retrospective analyses have shown that ${ }^{68} \mathrm{Ga}$-PSMA-11 PET imaging at the time of BCR leads to treatment changes in more than half of cases $(24,34)$. The treatment impact of ${ }^{68}$ Ga-PSMA-11 PET/ CT was prospectively evaluated in a multicenter study of 382 men with BCR, demonstrating management changes in $68 \%$ of cases-including $46 \%$ "major" changes, such as intermodality changes-based on the results of ${ }^{68} \mathrm{Ga}$ PSMA-11 imaging (35). In the afore-mentioned CONDOR 

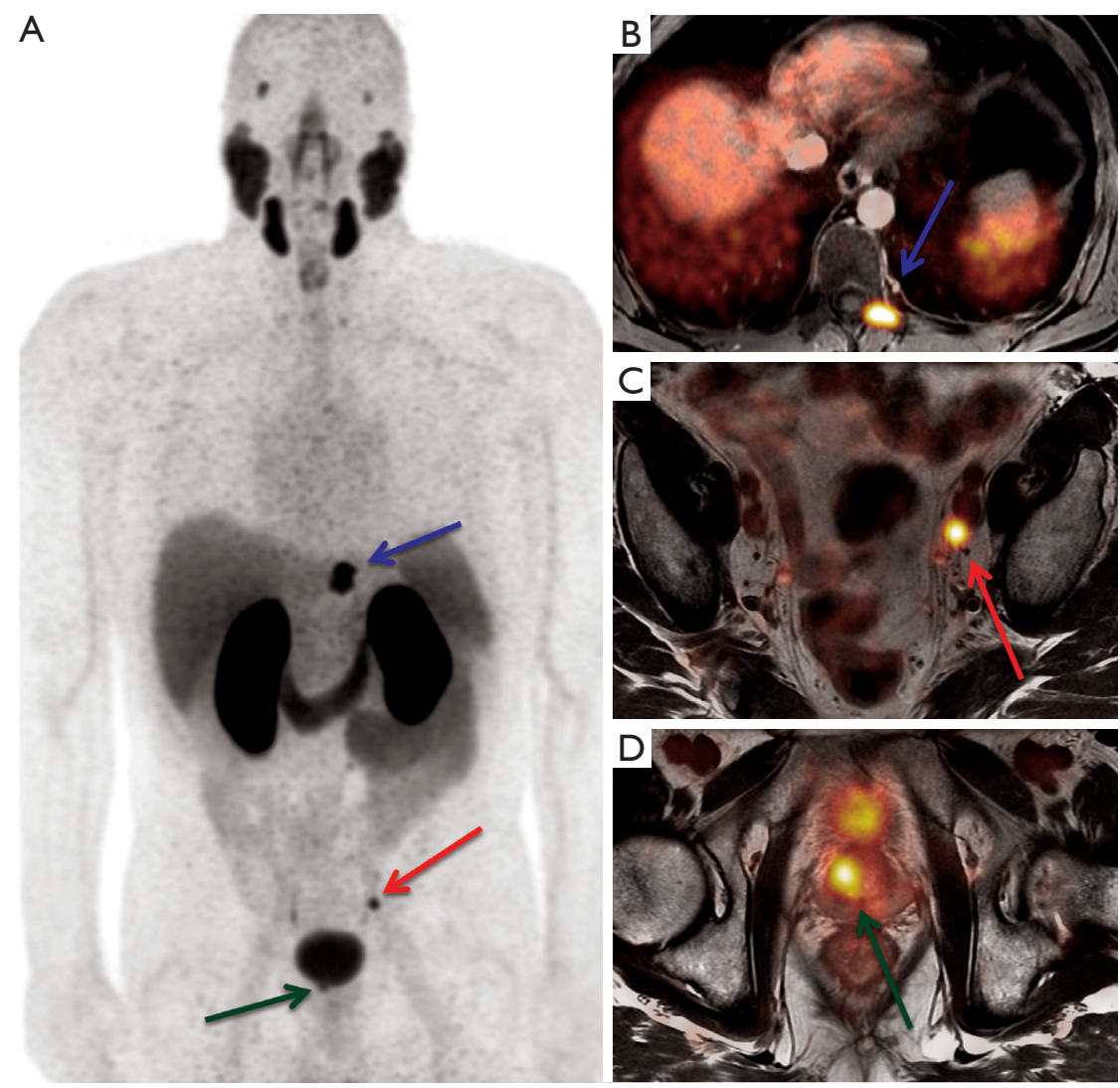

Figure $1{ }^{68} \mathrm{Ga}$-PSMA PET/MR in a patient with BCR. The patient developed a rising serum PSA level after prior RT for clinically localized PC. Serum PSA levels was up to $1.2 \mathrm{ng} / \mathrm{dL}$ at the time of imaging. The MIP (A) and axial fused PET/MR (B,C) images revealed locoregional recurrence in the prostatic fossa (green arrow) and a pelvic lymph node (red arrow), as well as distant metastatic disease in a thoracic vertebra (blue arrow). ${ }^{68}$ Ga-PSMA, gallium-68-labelled prostate-specific-membrane antigen; PET, positron-emission tomography; MR, magnetic resonance; BCR, biochemical recurrence; PSA, prostate specific antigen; PC, prostate cancer; MIP, maximal-intensity projection.

study, results of ${ }^{18} \mathrm{~F}$-DCFPyL PET/CT led to management changes for $64 \%$ of patients, including a change from systemic therapy to a curative treatment approach in $20 \%$ of cases (28). Whether such treatment changes will translate into improved patient outcomes remains to be determined, and is the focus of two ongoing clinical trials (NCT03762759, NCT03582774) (36).

Based on the above, current EAU guidelines recommend PSMA PET imaging in patients with BCR after prior RT fit for curative salvage therapy (strong recommendation) (25). PSMA PET imaging is also suggested in men with persistent or recurrent PSA elevation after RP (weak recommendation). European Association of Nuclear Medicine and Society of Nuclear Medicine and Molecular Imaging (EANM/SNMMI) guidelines similarly recommend use of PSMA imaging in patients with BCR eligible for salvage therapy—especially at low PSA values between 0.2 and $10 \mathrm{ng} / \mathrm{mL}(37)$.

\section{Molecular imaging for MDT}

Hellman and Weichselbaum (38) introduced the concept of an "oligometastatic" cancer state in 1995 to describe a subset of patients with limited systemic disease in which localized therapies may still achieve long-term survival. In PC, oligometastatic disease is variably defined by the presence of 3-5 or fewer metastases on conventional or molecular imaging. Although the optimal management of men with oligometastatic PC is not well established, there has been a growing interest in using MDT to improve outcomes or delay the start of androgen deprivation therapy (ADT). The SABR-COMET trial, which randomized 
patients with various tumor types (including 16 patients with PC) and oligometastatic disease on conventional imaging to standard-of-care palliative therapy with or without stereotactic ablative radiotherapy (SABR), showed a significant improvement in median overall survival (OS) (28 versus 41 months) with the addition of MDT (39). Given its increased sensitivity for early metastases, molecular imaging has been variously incorporated into trials of MDT in PC: in a prospective single center study of 57 patients with oligometastatic PC on PSMA PET imaging, Kneebone and colleague achieved a median biochemical disease-free survival of 11 months after treating visible lesions with stereotactic body RT (SBRT) and no systemic therapy (40). The phase 2 Observation versus stereotactic ablative RadiatIon for OLigometastatic prostate CancEr (ORIOLE) study randomized 54 patients with oligometastatic PC to MDT (i.e., SABR to all detected lesions) or observation alone. MDT was well-tolerated, and was associated with in a significant delay in progression (6-month progression rate of $19 \%$ versus $61 \%$ ) (41). Although oligometastatic disease in ORIOLE was defined using conventional imaging (3 or fewer metastases), pre-treatment PSMA PET imaging (using ${ }^{18} \mathrm{~F}$-DCFPyL) was performed in all patients assigned to the MDT arm. Treating physicians were blinded to the results of ${ }^{18} \mathrm{~F}$-DCFPyL imaging, and $44 \%$ of patients treated with MDT were retrospectively found to have additional PSMA-avid disease not included in the treatment fields. The 6-month progression rate in this subgroup was significantly higher than in patients with no untreated lesions (38\% versus 5\%).

While promising, these results should be interpreted with caution: systemic therapy with ADT remains the standard of care for patients with metastatic disease on conventional imaging (regardless of the number of lesions), with a preponderance of data supporting early treatment intensification with androgen-signaling inhibitors in metastatic hormone-sensitive PC (mHSPC). Men with oligometastatic disease that is not detected on conventional imaging may also benefit from systemic therapy, and prospective comparisons of MDT with ADT are still lacking. The role of MDT in patients with oligometastatic disease on PSMA PET imaging is the focus of several ongoing clinical trials, including a phase 2 trial (assessing the rate of undetectable PSA levels with combined antiandrogen therapy and SBRT in men with recurrent oligometastatic PC; NCT03902951), the randomized phase 2 STORM trial (comparing metastases-free survival in men with recurrent oligometastatic $\mathrm{PC}$ receiving ADT with
MDT with or without whole pelvic RT; NCT03569241), and the phase 3 ADOPT trial (comparing metastases PFS with MDT with or without short-term ADT in men with oligometastatic PC on PSMA PET; NCT04302454).

\section{PSMA PET in non-metastatic castration-resistant PC (nmCRPC)}

nmCRPC is defined by a rising serum PSA level despite castrate levels of testosterone, in the absence of metastatic disease on conventional imaging. When treated with ADT alone, nearly a third of these patients go on to develop distant metastases after 2 years. However, it was not until results of the SPARTAN trial were published in 2018-14 years after combined chemohormonal therapy was found to improve survival in $\mathrm{mCRPC}$ - that ADT intensification with apalutamide became established as a treatment option in nmCRPC (42). Since then, two additional agents (enzalutamide and darolutamide) have also been shown to improve metastasis-free survival in nmCRPC when combined with ADT.

In 2019, Fendler and colleagues performed a retrospective analysis of ${ }^{68} \mathrm{Ga}-\mathrm{PSMA}-11 \mathrm{PET} / \mathrm{CT}$ or PET/MRI in 200 patients with nmCRPC at high-risk for developing metastases-using enrollment criteria similar to those of the SPARTAN trial (43). PSMA-PET scan was positive in $98 \%$ of cases, and histopathologic confirmation $(n=30)$ yielded a patient-based PPV of $97 \%$. Fifty-five percent of patients were found to have distant metastases, while $44 \%$ had disease limited to the pelvis. Interestingly, oligometastatic disease (defined as 2-3 metastases) was seen in $14 \%$ of cases. These results largely support the current treatment paradigm of early ADT intensification in men with nmCRPC. Whether a subset of nmCRPC patients with oligometastatic disease may benefit from MDT has yet to be determined (Figure 2).

\section{PSMA PET in radioligand therapy (RLT) in MCRPC}

The impact of novel PET radiotracers on the management of PC extends well beyond the staging of early disease. In the era of personalized medicine, molecular-based imaging modalities can provide clinicians with a realtime, non-invasive assessment of cancer biology that is not affected by tumor heterogeneity. The emerging field of theranostics has capitalized on this insight by combining targeted therapies with companion imaging tracers to select patients and monitor treatment response. In vitro and 
A

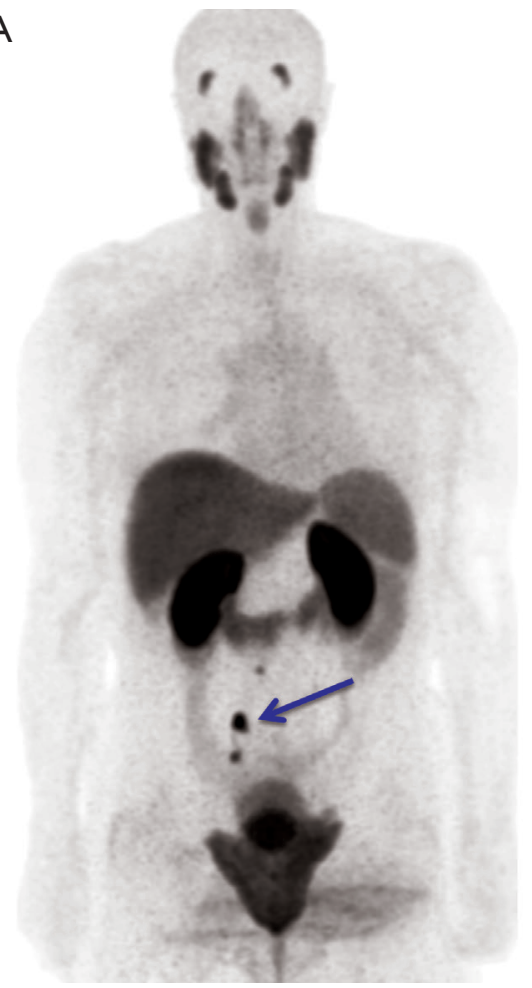

B

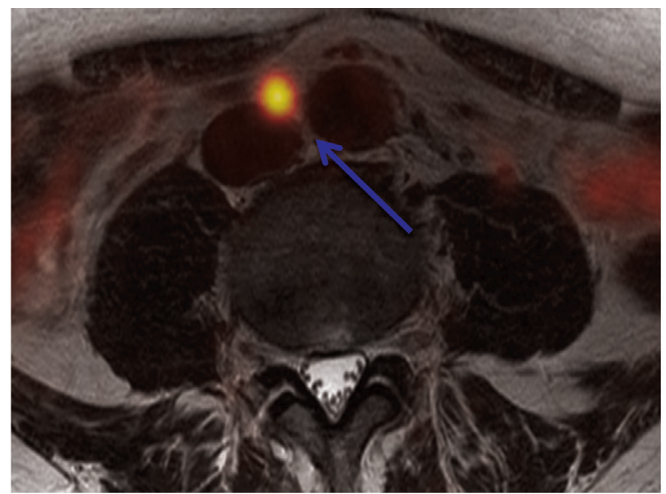

C

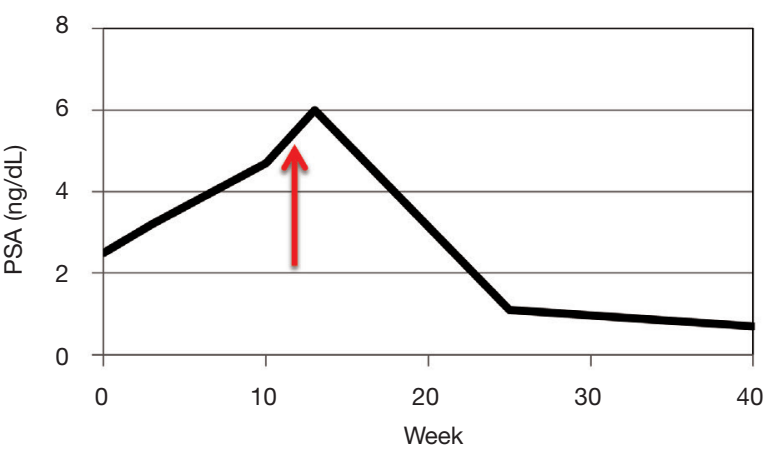

Figure $2{ }^{68} \mathrm{Ga}-\mathrm{PSMA}$ PET/MR in a patient with nmCRPC. The MIP (A) and axial fused PET/MR (B) images revealed oligometastatic disease with a PSMA-avid right iliac (blue arrow) and aortocaval lymph nodes. The patient underwent MDT with SBRT. Trend in serum PSA level over time (C) shows a greater than 50\% decrease in PSA after completion of MDT (red arrow). ${ }^{68}$ Ga-PSMA, gallium-68-labelled prostate-specific-membrane antigen; PET, positron-emission tomography; MR, magnetic resonance; nmCRPC, non-metastatic castrationresistant prostate cancer; MIP, maximal-intensity projection; MDT, metastasis-directed therapy; SBRT, stereotactic body radiation therapy; PSA, prostate specific antigen.

animal studies of PSMA-targeted metallic radionuclides began to appear in the early 2000s, and in 2005 Bander and colleagues published the first phase 1 study of PSMAtargeted RLT in men with PC (44). Additional phase 1 and 2 trials have established the efficacy of ${ }^{177} \mathrm{Lu}$ radiolabeled small molecules $(45,46)$ and antibodies $(47)$, in mCRPC, though the latter have been associated with a significantly higher rate of hematologic toxicities. Preliminary results of a randomized controlled trial of ${ }^{177} \mathrm{Lu}$-PSMA-617 versus cabazitaxel in mCRPC patients who had progressed after docetaxel (TheraP) have been recently published: with a median follow-up of 13 months, patients treated with PSMA RLT had significantly higher rates of PSA $\geq 50 \%$ response (66\% versus 37\%) and improved PFS (with a preliminary hazard ratio for death or progression of 0.69 ) (48). Grade 3 or higher thrombocytopenia occurred in $11 \%$ of patients treated with RLT. An international multicenter phase III trial (VISION) is ongoing.

Several studies have investigated the relationship between pre-treatment ${ }^{68} \mathrm{Ga}$-PSMA-11 PET uptake and treatment outcomes in patients receiving ${ }^{177} \mathrm{Lu}$ RLT: in one analysis of 30 patients with mCRPC, whole body tumor $\mathrm{SUV}_{\text {mean }}$ on pre-treatment ${ }^{68} \mathrm{Ga}$-PSMA-11 PET imaging predicted absorbed radiation dose on post-treatment quantitative single-photon emission computed tomography (SPECT)/ CT (49), which was in turn associated with PSA response at 12 weeks. In another phase 2 trial, pre-treatment PSMA SUV was predictive of $\geq 30 \%$ PSA reduction in patients receiving ${ }^{177} \mathrm{Lu}$ RLT (46). Grubmüller and colleagues also showed that on-treatment changes in ${ }^{68} \mathrm{Ga}$-PSMA-11 PET total tumor volume were predictive of PSA response and OS (50). Together these finding provide a rational for the use of ${ }^{68} \mathrm{Ga}$-PSMA-11 PET/CT to select patients for PSMA-targeted therapies, and those with low PSMA uptake 

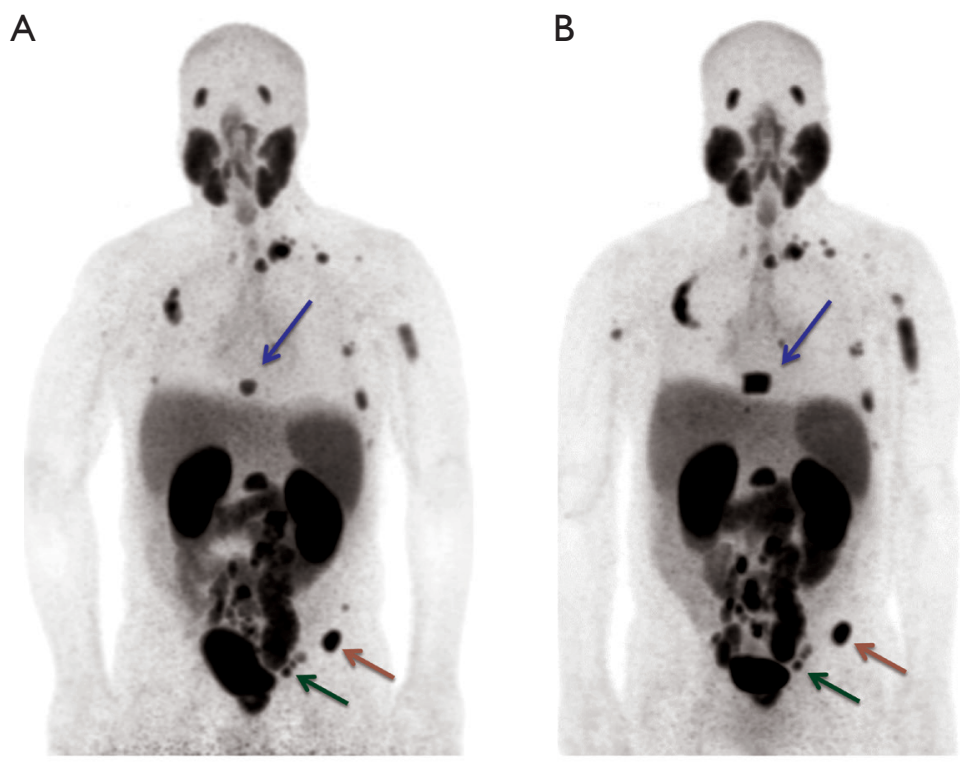

Figure $3{ }^{68} \mathrm{Ga}$-PSMA PET in a patient with de novo mHSPC. The pre-treatment MIP image (A) shows numerous nodal (blue and green arrows) and osseous (red arrow) metastases. ${ }^{68}$ Ga-PSMA PET was repeated 11 days after ADT initiation. On-treatment MIP image (B) shows increased radiotracer uptake in a nodal $\left(\mathrm{SUV}_{\max } 10\right.$ to 23, blue arrow) and osseous ( $\mathrm{SUV}_{\max } 25$ to 32, red arrow) metastasis. Another pelvic node metastasis had decreased uptake $\left(\mathrm{SUV}_{\max } 15\right.$ to 14 , green arrow) on ADT. The patient achieved a >90\% PSA response to ADT. ${ }^{68} \mathrm{Ga}$-PSMA, gallium-68-labelled prostate-specific-membrane antigen; PET, positron-emission tomography; mHSPC, metastatic hormonesensitive prostate cancer; MIP, maximal-intensity projection; ADT, androgen deprivation therapy; $\mathrm{SUV}_{\text {max }}$, maximum standardized uptake value; PSA, prostate specific antigen.

(generally defined as a tumor SUV max $_{\text {max }}$ less than 1 to 1.5 times that of the liver) have been excluded from clinical trials of PSMA RLT. Yet while patients with high PSMA uptake may derive the most benefit from PSMA-targeted therapy, the efficacy of RLT in an unselected patient population-or among patients with low PSMA expression-has not been fully investigated.

\section{PSMA PET in monitoring treatment response}

In addition to being used as companion diagnostic tool for novel targeted therapies, combined molecular and morphologic imaging modalities such as ${ }^{68}$ Ga-PSMA-11 PET have been applied to the monitoring of treatment response in patients receiving conventional PC therapies. Improved detection of metastatic disease may be particularly helpful in this setting to identify therapeutic failure early and minimize unnecessary treatment-related morbidity. Yet whereas PSMA is uniformly expressed in early PC, the characteristics of PSMA expression in advanced PC and the impact of prior or current therapies are less established.
Small, early studies of serial ${ }^{68} \mathrm{Ga}$-PSMA imaging after initiation of antiandrogen therapy have reported a flare phenomenon in a subset of patients (Figure 3) (51-54). Comparisons of pre- and on-treatment ${ }^{68} \mathrm{Ga}$-PSMA-11 $\mathrm{PET} / \mathrm{CT}$ scans 2-4 weeks after initiation of ADT or enzalutamide in 8 patients with metastatic PC showed an increase in PSMA uptake in more than half of casesdespite PSA-responses in all patients (52). This increase in PSMA uptake appears to peak 3-4 weeks after the start of antiandrogen therapy and does not seem to be mediated by a testosterone flare (54). A similar phenomenon has been observed in mCRPC patients starting androgen signaling inhibitors $(52,53)$. Although prospective validation in larger studies is needed, some investigators have sought to harness this transient increase in PSMA uptake to enhance response to PSMA-targeted RLT. The ongoing ENZA-p phase 2 trial will thus randomize $160 \mathrm{mCRPC}$ patients to enzalutamide with or without ${ }^{177} \mathrm{Lu}$-PSMA-617 (NCT04419402), while a recently published cohort of 10 men with mCRPC scheduled to undergo PMSAtargeted RLT showed a significant increase in PSMA uptake 
(without any significant change in PSA levels) after a short pre-treatment course of enzalutamide (55). Interestingly, 7 of the 10 patients included in this analysis had previously progressed on enzalutamide. Subsequent RLT outcomes are not yet available, and whether this strategy can meaningfully enhance response to ${ }^{177} \mathrm{Lu}$ RLT remains to be seen.

In comparison to these early changes in PSMA uptake, analyses of serial ${ }^{68} \mathrm{Ga}$-PSMA-11 PET imaging performed at longer time intervals have generally shown a correlation between treatment responses and decreased PSMA uptake, with no evidence of a flare phenomenon. In one retrospective analysis of 10 patients with metastatic hormone sensitive PC initiated on ADT, repeat ${ }^{68} \mathrm{Ga}$ PSMA-11 PET/CT performed after a median of 230 days on therapy showed decreased uptake in $71 \%$ of cases (56). All patients had a PSA response to androgen deprivation. Decreased PSMA uptake on serial PET imaging (performed at a median interval of 3 months) in a retrospective cohort of 26 mCRPC patients started on enzalutamide or abiraterone was perfectly associated with PSA or radiographic response (57). Another retrospective analysis of 43 patients undergoing 67 systemic therapies showed that on- or post-treatment changes in ${ }^{68} \mathrm{Ga}$-PSMA-11 SUV mean, $\mathrm{SUV}_{\text {max }}, \mathrm{SUV}_{\text {peak }}$ and total tumor volume were all associated with PSA response (58). Prospective validation will be needed to clarify the prognostic and predictive significance of ${ }^{68} \mathrm{Ga}$-PSMA uptake changes in patients receiving nonPSMA-targeted therapies.

\section{Limitations of PSMA PET imaging in PC}

PSMA PET imaging has integrated the standard of care in several countries outside of the US, and is expected to receive FDA approval in 2020. Yet while an extensive body of literature supports its use in PC, several limitations are worth highlighting.

Despite impressive patient-based sensitivities in men with BCR, ${ }^{68} \mathrm{Ga}$-PSMA-11 and ${ }^{18} \mathrm{~F}$-DCFPyL cannot reliably detect metastatic lymph nodes smaller than $5 \mathrm{~mm}(21,59-61)$. Consistent with these findings, most patients with BCR and a negative ${ }^{68} \mathrm{Ga}$-PSMA-11 PET/CT still achieve a PSA response to salvage pelvic radiation (62). Given that salvage radiotherapy is the current standard of care for men with a rising serum PSA level after a prior prostatectomy and no evidence of distant metastases, the ability to localize pelvic recurrence may be less clinically relevant than the ability to rule out distant disease. Yet whether the sensitivity of PSMA-based imaging for metastatic disease at very low serum PSA levels is sufficient to improve patient outcomes in BCR still needs to be determined.

Second, the role of ${ }^{68} \mathrm{Ga}$-PSMA and other molecularbased imaging modalities in metastatic PC is far less established than in patients with clinically localized or biochemically recurrent disease. Because of its improved sensitivity PSMA imaging often leads to upstaging of tumor burden $(21,23)$; however, most therapeutic trials still rely on conventional imaging to characterize their patients' volume of disease. As a result, clinicians are increasingly faced with discordant staging information across conventional and molecular-based imaging modalities. However, outside of PSMA-targeted RLT PSMA PET/CT has not yet been shown to impact treatment outcomes in PC and management decisions based on molecular imaging alone should be made with caution. Furthermore, the effect of different therapies on PSMA uptake is still poorly understood. Although attempts have been made to harness treatment-induced PSMA flares to improve the diagnostic performance of PSMA imaging, significant intra- and interpatient heterogeneity is likely to limit the clinical impact of such approaches $(52,54)$.

Finally, despite its name, PSMA expression is not specific to prostatic epithelium. Mild uptake can be seen in benign osseous conditions such as fibrous dysplasia, fractures or fibrous osseous defects (63). PSMA expression has also been reported on the neovasculature of several non-prostatic malignancies including renal cell carcinoma, hepatocellular carcinoma, thyroid cancers and gliomas (64). Conversely, loss of PSMA uptake has been described in a subset of patients with advanced metastatic PC. Several cases of treatment-emergent small cell neuroendocrine carcinoma (t-SCNC) presenting as PSMA negative lesions on PET/ CT have been reported in the literature $(65,66)$, and around $20 \%$ to $25 \%$ of mCRPC patients are excluded from clinical trials of ${ }^{177} \mathrm{Lu}$ RLT due to uniformly low PSMA uptake (Figure 4) or lesions with discordant low PSMA and high ${ }^{18} \mathrm{FDG}$-uptake $(46,67)$. While the histologic and genomic features associated with loss of PSMA expression in $\mathrm{mCRPC}$ have not been described, the finding of low or heterogeneous uptake on PSMA PET imaging appears to be associated with poor clinical outcomes (67). Although further studies will be needed to clarify the prognostic and predictive significance of PSMA expression in advanced PC, the finding of low or absent PSMA-uptake in a subset of tumors is likely to limit the use of PSMA-based PET/CT as a standalone imaging modality in metastatic PC. However, variations in PSMA uptake may also provide key molecular 

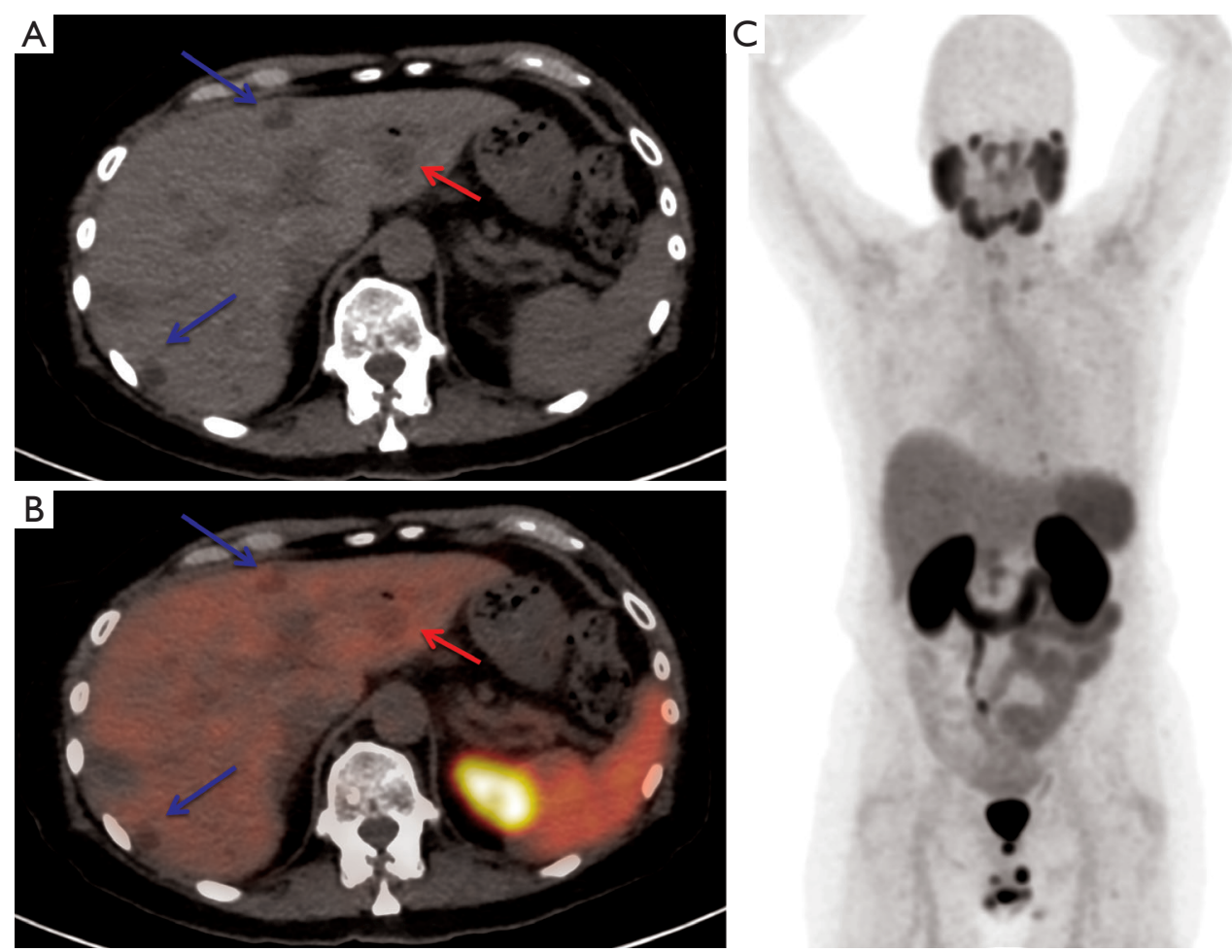

Figure $4{ }^{68} \mathrm{Ga}$-PSMA PET/CT in a patient with mCRPC with low PSMA uptake. The axial CT (A) shows multiple hypodense hepatic lesions consistent with PC metastases (blue and red arrows). Axial fused PET/CT (B) and MIP (C) images show no corresponding PSMAavid lesion. The patient underwent an imaging-guided biopsy of a lesion in the left hepatic lobe (red arrow), which revealed prostatic adenocarcinoma with AR amplification and a TMPRSS2:ERG gene fusion. ${ }^{68}$ Ga-PSMA, gallium-68-labelled prostate-specific-membrane antigen; PET, positron-emission tomography; CT, computed tomography; mCRPC, metastatic castration-resistant prostate cancer; PC, prostate cancer; MIP, maximal-intensity projection; AR, androgen receptor; TMPRSS2-ERG, transmembrane protease serine 2:v-ets erythroblastosis virus E26 oncogene homolog.

insight on underlying tumor biology; by helping to identify more aggressive variants (such as t-SCNC) PSMA imaging may yet still play an important role in the monitoring patients with advanced PC.

\section{Conclusions}

Several novel radiotracers have been investigated over the past two decades to address the limitations of conventional imaging modalities in the diagnosis and monitoring of PC. PSMA-based radiotracers such as ${ }^{68} \mathrm{Ga}$-PSMA- 11 have been the most widely studied. There is now an extensive body of literature to support their use in clinically localized and BCR PC and when available, PSMA-based imaging has been shown to affect treatment decisions in these settings. Improved PC detection has also helped to identify a subset of patients with oligometastatic disease who may benefit from MDT in addition (or as an alternative) to conventional systemic therapies. Ongoing prospective studies will begin to clarify the impact of PSMA imaging on patient outcomes.

In comparison to clinically localized PC and BCR, the role of PSMA-based imaging in metastatic PC is less established. Higher PSMA uptake has been shown to correlate with PSA response to PSMA-targeted RLT. However, nearly a quarter of patients with mCRPC fail current RLT screening criteria due to low or heterogeneous PSMA uptake. As PSMA-based imaging becomes increasingly incorporated into clinical practice, there is an urgent need to determine the optimal the management of patients with low-PSMA uptake-and the role of PSMAtargeted RLT in this population. Similarly, the effect of hormonal or cytotoxic chemotherapies on PSMA uptake will need to be clarified. Finally, unlike conventional imaging, combined morphologic and molecular based 
modalities such as PSMA PET have the potential to offer a non-invasive assessment of underlying tumor biology. Although loss of PSMA uptake in a subset of mCRPC patients may prevent the use of PSMA imaging as a standalone imaging modality, it may also provide clinically valuable molecular insight and help identify more aggressive PC variants. Further studies will be needed to define the underlying mechanisms, and the prognostic implications of PSMA uptake patterns in advanced PC.

\section{Acknowledgments}

Funding: None.

\section{Footnote}

Provenance and Peer Review: This article was commissioned by the Guest Editors (Badrinath R. Konety, Daniel W. Lin) for the series "Current and Future Topics on Prostate Cancer" published in Translational Andrology and Urology. The article has undergone external peer review.

Reporting Checklist: The authors have completed the NARRATIVE REVIEW reporting checklist. Available at http://dx.doi.org/10.21037/tau-20-1047

Peer Review File: Available at http://dx.doi.org/10.21037/tau20-1047

Conflicts of Interest: All authors have completed the ICMJE uniform disclosure form (available at http://dx.doi. org/10.21037/tau-20-1047). The series "Current and Future Topics on Prostate Cancer" was commissioned by the editorial office without any funding or sponsorship. Dr. RA reports grants from Janssen, grants and personal fees from Merck, grants and personal fees from AstraZeneca, personal fees from Clovis Oncology, personal fees from Dendreon, outside the submitted work. Dr. TH reports personal fees from Blue Earth, personal fees from Progenics, grants from Advanced Accelerator Applications, personal fees from Curium, outside the submitted work. The other author has no other conflicts of interest to declare.

Ethical Statement: The authors are accountable for all aspects of the work in ensuring that questions related to the accuracy or integrity of any part of the work are appropriately investigated and resolved.
Open Access Statement: This is an Open Access article distributed in accordance with the Creative Commons Attribution-NonCommercial-NoDerivs 4.0 International License (CC BY-NC-ND 4.0), which permits the noncommercial replication and distribution of the article with the strict proviso that no changes or edits are made and the original work is properly cited (including links to both the formal publication through the relevant DOI and the license). See: https://creativecommons.org/licenses/by-nc-nd/4.0/.

\section{References}

1. Siegel RL, Miller KD, Jemal A: Cancer statistics, 2019. CA Cancer J Clin 2019;69:7-34.

2. Hövels AM, Heesakkers RM, Adang EM, et al. The diagnostic accuracy of CT and MRI in the staging of pelvic lymph nodes in patients with prostate cancer: a metaanalysis. Clin Radiol 2008;63:387-95.

3. Padhani AR, Lecouvet FE, Tunariu N, et al. Rationale for Modernising Imaging in Advanced Prostate Cancer. Eur Urol Focus 2017;3:223-39.

4. Hofer C, Laubenbacher C, Block T, et al. Fluorine-18fluorodeoxyglucose positron emission tomography is useless for the detection of local recurrence after radical prostatectomy. Eur Urol 1999;36:31-5.

5. Liu IJ, Zafar MB, Lai YH, et al. Fluorodeoxyglucose positron emission tomography studies in diagnosis and staging of clinically organ-confined prostate cancer. Urology 2001;57:108-11.

6. Picchio M, Briganti A, Fanti S, et al. The role of choline positron emission tomography/computed tomography in the management of patients with prostate-specific antigen progression after radical treatment of prostate cancer. Eur Urol 2011;59:51-60.

7. Krause BJ, Souvatzoglou M, Tuncel M, et al. The detection rate of $[11 \mathrm{C}]$ choline-PET/CT depends on the serum PSA-value in patients with biochemical recurrence of prostate cancer. Eur J Nucl Med Mol Imaging 2008;35:18-23.

8. Chondrogiannis S, Marzola MC, Ferretti A, et al. Role of $18 \mathrm{~F}$-choline PET/CT in suspicion of relapse following definitive radiotherapy for prostate cancer. Eur J Nucl Med Mol Imaging 2013;40:1356-64.

9. Cimitan M, Bortolus R, Morassut S, et al. [18F] fluorocholine PET/CT imaging for the detection of recurrent prostate cancer at PSA relapse: experience in 100 consecutive patients. Eur J Nucl Med Mol Imaging 
2006;33:1387-98.

10. Nanni C, Zanoni L, Pultrone C, et al. (18)F-FACBC (anti1-amino-3-(18)F-fluorocyclobutane-1-carboxylic acid) versus (11)C-choline PET/CT in prostate cancer relapse: results of a prospective trial. Eur J Nucl Med Mol Imaging 2016;43:1601-10.

11. Bin X, Yong S, Kong QF, et al. Diagnostic Performance of PET/CT Using 18F-FACBC in Prostate Cancer: A MetaAnalysis. Front Oncol 2020;9:1438.

12. Armstrong JM, Martin CR, Dechet C, et al. 18F-fluciclovine PET CT detection of biochemical recurrent prostate cancer at specific PSA thresholds after definitive treatment. Urol Oncol 2020;38:636.e1-636.e6.

13. Annunziata S, Pizzuto DA, Treglia G. Diagnostic Performance of PET Imaging Using Different Radiopharmaceuticals in Prostate Cancer According to Published Meta-Analyses. Cancers (Basel) 2020;12:2153.

14. Suzuki H, Inoue Y, Fujimoto H, et al. Diagnostic performance and safety of NMK36 (trans-1-amino-3-[18F] fluorocyclobutanecarboxylic acid)-PET/CT in primary prostate cancer: multicenter Phase IIb clinical trial. Jpn J Clin Oncol 2016;46:152-62.

15. Silver DA, Pellicer I, Fair WR, et al. Prostate-specific membrane antigen expression in normal and malignant human tissues. Clin Cancer Res 1997;3:81-5.

16. Giesel FL, Hadaschik B, Cardinale J, et al. F-18 labelled PSMA-1007: biodistribution, radiation dosimetry and histopathological validation of tumor lesions in prostate cancer patients. Eur J Nucl Med Mol Imaging 2017;44:678-88.

17. Afshar-Oromieh A, Malcher A, Eder M, et al. PET imaging with a [68Ga]gallium-labelled PSMA ligand for the diagnosis of prostate cancer: biodistribution in humans and first evaluation of tumour lesions. Eur J Nucl Med Mol Imaging 2013;40:486-95.

18. Maurer T, Gschwend JE, Rauscher I, et al. Diagnostic Efficacy of (68)Gallium-PSMA Positron Emission Tomography Compared to Conventional Imaging for Lymph Node Staging of 130 Consecutive Patients with Intermediate to High Risk Prostate Cancer. J Urol 2016;195:1436-43.

19. Hope TA, Goodman JZ, Allen IE, et al. Metaanalysis of 68Ga-PSMA-11 PET Accuracy for the Detection of Prostate Cancer Validated by Histopathology. J Nucl Med 2019;60:786-93.

20. van Kalmthout LWM, van Melick HHE, Lavalaye J, et al. Prospective Validation of Gallium-68 Prostate Specific Membrane Antigen-Positron Emission Tomography/
Computerized Tomography for Primary Staging of Prostate Cancer. J Urol 2020;203:537-45.

21. Rowe S, Gorin M, Pienta K, et al. Results from the OSPREY trial: A PrOspective Phase 2/3 Multi-Center Study of 18F-DCFPyL PET/CT Imaging in Patients with PRostate Cancer - Examination of Diagnostic AccuracY. J Nucl Med 2019;60:586.

22. Jansen BHE, Bodar YJL, Zwezerijnen GJC, et al. Pelvic lymph-node staging with 18F-DCFPyL PET/CT prior to extended pelvic lymph-node dissection in primary prostate cancer - the SALT trial. Eur J Nucl Med Mol Imaging 2021;48:509-20.

23. Hofman MS, Lawrentschuk N, Francis RJ, et al. Prostatespecific membrane antigen PET-CT in patients with high-risk prostate cancer before curative-intent surgery or radiotherapy (proPSMA): a prospective, randomised, multicentre study. Lancet 2020;395:1208-16.

24. Roach PJ, Francis R, Emmett L, et al. The Impact of 68Ga-PSMA PET/CT on Management Intent in Prostate Cancer: Results of an Australian Prospective Multicenter Study. J Nucl Med 2018;59:82-8.

25. Mottet N, Cornford P, van den Bergh RCN, et al. EAU Guidelines. 2020. Available online: https://uroweb.org/ guideline/prostate-cancer/

26. Afshar-Oromieh A, Holland-Letz T, Giesel FL, et al. Diagnostic performance of 68Ga-PSMA-11 (HBEDCC) PET/CT in patients with recurrent prostate cancer: evaluation in 1007 patients. Eur J Nucl Med Mol Imaging 2017;44:1258-68.

27. Fendler WP, Calais J, Eiber M, et al. Assessment of 68GaPSMA-11 PET Accuracy in Localizing Recurrent Prostate Cancer: A Prospective Single-Arm Clinical Trial. JAMA Oncol 2019;5:856-63.

28. Morris MJ, Carroll PR, Saperstein L, et al. Impact of PSMA-targeted imaging with 18F-DCFPyL-PET/CT on clinical management of patients (pts) with biochemically recurrent (BCR) prostate cancer $(\mathrm{PCa})$ : Results from a phase III, prospective, multicenter study (CONDOR). J Clin Oncol 2020;38:abstr 5501.

29. Treglia G, Annunziata S, Pizzuto DA, et al. Detection Rate of 18F-Labeled PSMA PET/CT in Biochemical Recurrent Prostate Cancer: A Systematic Review and a Meta-Analysis. Cancers (Basel) 2019;11:710.

30. Perera M, Papa N, Roberts M, et al. Gallium-68 Prostate-specific Membrane Antigen Positron Emission Tomography in Advanced Prostate Cancer-Updated Diagnostic Utility, Sensitivity, Specificity, and Distribution of Prostate-specific Membrane Antigen-avid Lesions: 
A Systematic Review and Meta-analysis. Eur Urol 2020;77:403-17.

31. Treglia G, Pereira Mestre R, Ferrari M, et al. Radiolabelled choline versus PSMA PET/CT in prostate cancer restaging: a meta-analysis. Am J Nucl Med Mol Imaging 2019;9:127-39.

32. Calais J, Ceci F, Eiber M, et al. 18F-fluciclovine PETCT and 68Ga-PSMA-11 PET-CT in patients with early biochemical recurrence after prostatectomy: a prospective, single-centre, single-arm, comparative imaging trial. Lancet Oncol 2019;20:1286-94.

33. Calais J, Czernin J, Cao M, et al. 68Ga-PSMA-11 PET/ CT Mapping of Prostate Cancer Biochemical Recurrence After Radical Prostatectomy in 270 Patients with a PSA Level of Less Than $1.0 \mathrm{ng} / \mathrm{mL}$ : Impact on Salvage Radiotherapy Planning. J Nucl Med 2018;59:230-7.

34. Han S, Woo S, Kim YJ, et al. Impact of 68Ga-PSMA PET on the Management of Patients with Prostate Cancer: A Systematic Review and Meta-analysis. Eur Urol 2018;74:179-90.

35. Fendler WP, Ferdinandus J, Czernin J, et al. Impact of 68Ga-PSMA-11 PET on the Management of Recurrent Prostate Cancer in a Prospective Single-Arm Clinical Trial. J Nucl Med 2020;61:1793-9.

36. Calais J, Czernin J, Fendler WP, et al. Randomized prospective phase III trial of 68Ga-PSMA-11 PET/CT molecular imaging for prostate cancer salvage radiotherapy planning [PSMA-SRT]. BMC Cancer 2019;19:18.

37. Fendler WP, Eiber M, Beheshti M, et al. 68Ga-PSMA PET/CT: Joint EANM and SNMMI procedure guideline for prostate cancer imaging: version 1.0. Eur J Nucl Med Mol Imaging 2017;44:1014-24.

38. Hellman S, Weichselbaum RR. Oligometastases. J Clin Oncol 1995;13:8-10.

39. Palma DA, Olson R, Harrow S, et al. Stereotactic ablative radiotherapy versus standard of care palliative treatment in patients with oligometastatic cancers (SABRCOMET): a randomised, phase 2, open-label trial. Lancet 2019;393:2051-8.

40. Kneebone A, Hruby G, Ainsworth H, et al. Stereotactic Body Radiotherapy for Oligometastatic Prostate Cancer Detected via Prostate-specific Membrane Antigen Positron Emission Tomography. Eur Urol Oncol 2018;1:531-7.

41. Phillips R, Shi WY, Deek M, et al. Outcomes of Observation vs Stereotactic Ablative Radiation for Oligometastatic Prostate Cancer: The ORIOLE Phase 2 Randomized Clinical Trial. JAMA Oncol 2020;6:650-9. 42. Smith MR, Saad F, Chowdhury S, et al. Apalutamide
Treatment and Metastasis-free Survival in Prostate Cancer. N Engl J Med 2018;378:1408-18.

43. Fendler WP, Weber M, Iravani A, et al. ProstateSpecific Membrane Antigen Ligand Positron Emission Tomography in Men with Nonmetastatic Castration-Resistant Prostate Cancer. Clin Cancer Res 2019;25:7448-54.

44. Bander NH, Milowsky MI, Nanus DM, et al. Phase I trial of 177lutetium-labeled J591, a monoclonal antibody to prostate-specific membrane antigen, in patients with androgen-independent prostate cancer. J Clin Oncol 2005;23:4591-601.

45. Hofman MS, Violet J, Hicks RJ, et al. [177Lu]-PSMA-617 radionuclide treatment in patients with metastatic castration-resistant prostate cancer (LuPSMA trial): a single-centre, single-arm, phase 2 study. Lancet Oncol 2018;19:825-33.

46. Emmett L, Crumbaker M, Ho B, et al. Results of a Prospective Phase 2 Pilot Trial of 177Lu-PSMA-617 Therapy for Metastatic Castration-Resistant Prostate Cancer Including Imaging Predictors of Treatment Response and Patterns of Progression. Clin Genitourin Cancer 2019;17:15-22.

47. Tagawa ST, Milowsky MI, Morris M, et al. Phase II study of Lutetium-177-labeled anti-prostate-specific membrane antigen monoclonal antibody J591 for metastatic castration-resistant prostate cancer. Clin Cancer Res 2013;19:5182-91.

48. Hofman MS, Emmett L, Sandhu SK, et al. TheraP: A randomised phase II trial of 177Lu-PSMA-617 (LuPSMA) theranostic versus cabazitaxel in metastatic castration resistant prostate cancer (mCRPC) progressing after docetaxel: Initial results (ANZUP protocol 1603). J Clin Oncol 2020;38:abstr 5500.

49. Violet J, Jackson P, Ferdinandus J, et al. Dosimetry of 177Lu-PSMA-617 in Metastatic Castration-Resistant Prostate Cancer: Correlations Between Pretherapeutic Imaging and Whole-Body Tumor Dosimetry with Treatment Outcomes. J Nucl Med 2019;60:517-23.

50. Grubmüller B, Senn D, Kramer G, et al. Response assessment using 68Ga-PSMA ligand PET in patients undergoing 177Lu-PSMA radioligand therapy for metastatic castration-resistant prostate cancer. Eur J Nucl Med Mol Imaging 2019;46:1063-72.

51. Hope TA, Truillet C, Ehman EC, et al. 68Ga-PSMA-11 PET Imaging of Response to Androgen Receptor Inhibition: First Human Experience. J Nucl Med 2017;58:81-4. 
52. Aggarwal R, Wei X, Kim W, et al. Heterogeneous Flare in Prostate-specific Membrane Antigen Positron Emission Tomography Tracer Uptake with Initiation of Androgen Pathway Blockade in Metastatic Prostate Cancer. Eur Urol Oncol 2018;1:78-82.

53. Emmett L, Yin C, Crumbaker M, et al. Rapid Modulation of PSMA Expression by Androgen Deprivation: Serial 68Ga-PSMA-11 PET in Men with Hormone-Sensitive and Castrate-Resistant Prostate Cancer Commencing Androgen Blockade. J Nucl Med 2019;60:950-4.

54. Ettala O, Malaspina S, Tuokkola T, et al. Prospective study on the effect of short-term androgen deprivation therapy on PSMA uptake evaluated with 68Ga-PSMA-11 PET/ MRI in men with treatment-naïve prostate cancer. Eur J Nucl Med Mol Imaging 2020;47:665-73.

55. Rosar F, Dewes S, Ries M, et al. New insights in the paradigm of upregulation of tumoral PSMA expression by androgen receptor blockade: Enzalutamide induces PSMA upregulation in castration-resistant prostate cancer even in patients having previously progressed on enzalutamide. Eur J Nucl Med Mol Imaging 2020;47:687-94.

56. Afshar-Oromieh A, Debus N, Uhrig M, et al. Impact of long-term androgen deprivation therapy on PSMA ligand PET/CT in patients with castration-sensitive prostate cancer. Eur J Nucl Med Mol Imaging 2018;45:2045-54.

57. Plouznikoff N, Artigas C, Sideris S, et al. Evaluation of PSMA expression changes on PET/CT before and after initiation of novel antiandrogen drugs (enzalutamide or abiraterone) in metastatic castration-resistant prostate cancer patients. Ann Nucl Med 2019;33:945-54.

58. Grubmüller B, Rasul S, Baltzer P, et al. Response assessment using [68 Ga]Ga-PSMA ligand PET in patients undergoing systemic therapy for metastatic castrationresistant prostate cancer. Prostate. 2020;80:74-82.

59. van Leeuwen PJ, Emmett L, Ho B, et al. Prospective evaluation of 68 Gallium-prostate-specific membrane antigen positron emission tomography/computed tomography for preoperative lymph node staging in

Cite this article as: de Kouchkovsky I, Aggarwal R, Hope TA. Prostate-specific membrane antigen (PSMA)-based imaging in localized and advanced prostate cancer: a narrative review. Transl Androl Urol 2021;10(7):3130-3143. doi: 10.21037/tau-201047 prostate cancer. BJU Int 2017;119:209-15.

60. Budäus L, Leyh-Bannurah SR, Salomon G, et al. Initial Experience of (68)Ga-PSMA PET/CT Imaging in High-risk Prostate Cancer Patients Prior to Radical Prostatectomy. Eur Urol 2016;69:393-6.

61. Öbek C, Doğanca T, Demirci E, et al. The accuracy of 68Ga-PSMA PET/CT in primary lymph node staging in high-risk prostate cancer. Eur J Nucl Med Mol Imaging 2017;44:1806-12.

62. Emmett L, van Leeuwen PJ, Nandurkar R, et al. Treatment Outcomes from 68Ga-PSMA PET/CTInformed Salvage Radiation Treatment in Men with Rising PSA After Radical Prostatectomy: Prognostic Value of a Negative PSMA PET. J Nucl Med 2017;58:1972-76.

63. de Galiza Barbosa F, Queiroz MA, Nunes RF, et al. Nonprostatic diseases on PSMA PET imaging: a spectrum of benign and malignant findings. Cancer Imaging 2020;20:23.

64. Van de Wiele C, Sathekge M, de Spiegeleer B, et al. PSMA-Targeting Positron Emission Agents for Imaging Solid Tumors Other Than Non-Prostate Carcinoma: A Systematic Review. Int J Mol Sci 2019;20:4886.

65. Tosoian JJ, Gorin MA, Rowe SP, et al. Correlation of PSMA-Targeted 18F-DCFPyL PET/CT Findings With Immunohistochemical and Genomic Data in a Patient With Metastatic Neuroendocrine Prostate Cancer. Clin Genitourin Cancer 2017;15:e65-8.

66. Chakraborty PS, Tripathi M, Agarwal KK, et al. Metastatic poorly differentiated prostatic carcinoma with neuroendocrine differentiation: negative on 68Ga-PSMA PET/CT. Clin Nucl Med 2015;40:e163-6.

67. Thang SP, Violet J, Sandhu S, et al. Poor Outcomes for Patients with Metastatic Castration-resistant Prostate Cancer with Low Prostate-specific Membrane Antigen (PSMA) Expression Deemed Ineligible for 177Lulabelled PSMA Radioligand Therapy. Eur Urol Oncol 2019;2:670-6. 